\begin{tabular}{|c|l|}
\hline Title & Paradoxical effects of chondroitin sulfate E on Japanese encephalitis viral infection \\
\hline Author(s) & $\begin{array}{l}\text { Kim, Eunmi; Okumura, Megumi; Sawa, Hirofumi; Miy azaki, Tadaaki; Fujikura, Dai suke; Y amada, Shuhei; Sugahara, } \\
\text { Kazuyuki; Sasaki, Michihito; Kimura, Takashi }\end{array}$ \\
\hline Citation & $\begin{array}{l}\text { Biochemical and Biophysical Research Communications, 409(4), 717-722 } \\
\text { https://doi.org/10.1016/.bbrc.2011.05.072 }\end{array}$ \\
\hline Issue Date & 2011-06-17 \\
\hline Doc URL & http://hdl.handle.net/2115/46843 \\
\hline Type & article (author version) \\
\hline File Information & BBC409-4_717-722.pdf \\
\hline
\end{tabular}

Instructions for use 


\section{Paradoxical effects of chondroitin sulfate-E on Japanese encephalitis viral infection}

Eunmi Kim ${ }^{1}$, Megumi Okumura ${ }^{1}$, Hirofumi Sawa ${ }^{1,4}$, Tadaaki Miyazaki ${ }^{2}$, Daisuke Fujikura ${ }^{2}$, Shuhei Yamada ${ }^{3}$, Kazuyuki Sugahara ${ }^{3}$, Michihito Sasaki ${ }^{1}$, and Takashi Kimura ${ }^{{ }^{*}}$

Department of Molecular Pathobiology, ${ }^{1}$ and Bioresources, ${ }^{2}$ Hokkaido University Research Center for Zoonosis Control, Sapporo 001-0020, Japan, Laboratory of Proteoglycan Signaling and Therapeutics, Graduate School of Life Science, Frontier Research Center for Post-Genomic Science and Technology, Hokkaido University, Sapporo 001-0021, Japan, ${ }^{3}$ and Global COE Program, Hokkaido University, Sapporo, Japan ${ }^{4}$

\section{*Corresponding author}

Fax: +81 11706 7370. E-mail address: kimura@czc.hokudai.ac.jp 


\begin{abstract}
Glycosaminoglycans (GAGs) have diverse functions in the body and are involved in viral infection. The purpose of this study was to evaluate the possible roles of the E-disaccharide units GlcA $\beta 1$-3GalNAc(4,6-O-disulfate) of chondroitin sulfate (CS), a GAG involved in neuritogenesis and neuronal migration, in Japanese encephalitis virus (JEV) infection. Soluble CS-E (sCS-E) derived from squid cartilage inhibited JEV infection in African green monkey kidney-derived Vero cells and baby hamster kidney-derived BHK cells by interfering with viral attachment. In contrast, sCS-E enhanced viral infection in the mouse neuroblastoma cell line Neuro-2a, despite the fact that viral attachment to Neuro-2a cells was inhibited by sCS-E. This enhancement effect in Neuro-2a cells seemed to be related to increased viral RNA replication and was also observed in a rat infection model in which intracerebral coadministration of sCS-E with JEV in 17-day-old rats resulted in higher brain viral loads than in rats infected without sCS-E administration. These results show the paradoxical effects of sCS-E on JEV infection in different cell types and indicate that potential use of sCS-E as an antiviral agent against JEV infection should be approached with caution considering its effects in the neuron, the major target of JEV.
\end{abstract}

Keyword: chondroitin sulfate E, Japanese encephalitis virus, neural cell 


\section{Introduction}

Japanese encephalitis virus (JEV) is a member of the Flaviviridae family that is transmitted primarily by Culex tritaeniorhynchus mosquitoes. Although most JEV infections in humans are asymptomatic, clinical cases tend to manifest as severe, often fatal encephalitis. Neurons are the major target of JEV in the brain [1].

Proteoglycans (PGs), which are composed of glycosaminoglycans (GAGs) and core proteins, are major components of the cell surface and the extracellular matrix. Chondroitin sulfate (CS)-PG constitutes the major population of GAGs in the central nervous system (CNS). CS-PG plays important roles in the developing and adult CNS with respect to the regulation of cell proliferation, differentiation, neuronal migration, neurite extension, neural plasticity, and axonal regeneration [2].

Recently, many researchers have been interested in the relationships between GAGs and viral infection. Some viruses use cell-surface PGs as their receptors [3-7], and virus clearance involves extracellular and cell surface PGs in the bloodstream [8]. However, the role of CS in flavivirus infection, especially within the CNS, remains uncertain.

The purpose of this study was to investigate the influence of soluble CS-E rich in GlcA $\beta 1$-3GalNAc(4,6-O-disulfate) unit, on JEV infection in a neural cell line and in animal brains. 


\section{Material and methods}

Cells, viruses, and reagents. Neuro-2a (mouse neuroblastoma) cells were grown in high-glucose Dulbecco's Modified Eagle Medium (DMEM) (Sigma, St. Louis, MO) supplemented with 10\% fetal calf serum (FCS). Baby hamster kidney (BHK-21) cells were grown in DMEM supplemented with 10\% FCS. African green monkey kidney (Vero) cells were grown in Minimum Essential Medium (Nissui, Tokyo, Japan) supplemented with 5\% FCS. The JEV Nakayama strain was kindly provided by Dr. Ikuo Takashima (Hokkaido University, Sapporo, Japan) and was cloned by 3 rounds of plaque purification. Soluble CS (sCS) -A, -B, -C, -D, and -E were purchased from Seikagaku Corp. (Tokyo, Japan). Heparin was purchased from Sigma (St. Louis, MO)

Immunofluorescence. Cells were fixed with methanol-acetone $(1: 1, \mathrm{v} / \mathrm{v})$ for $30 \mathrm{~min}$, washed with phosphate-buffered saline (PBS), and blocked with $2 \%$ bovine serum albumin (BSA)-PBS. Cells were incubated with rabbit anti-JEV serum in 1\% BSA-PBS at room temperature for $30 \mathrm{~min}$, followed by incubation with Alexa-594-conjugated anti-rabbit IgG (Invitrogen, Carlsbad, CA). The cell nuclei were counterstained with DAPI (4,6-diamidino-2-phenylindole). Fluorescence was observed with an inverted fluorescence microscope (IX70, Olympus, Tokyo, Japan). The number of JEV antigen-positive cells was counted by using MetaMorph software (MDS Analytical Technologies, Toronto, Canada).

Focus reduction assay. A focus reduction assay was performed as described by Aoki et [9] with modifications. Briefly, 40 focus-forming units (FFU) of JEV were premixed with 10-fold serial dilutions of sCS-E and incubated at $4{ }^{\circ} \mathrm{C}$ for $30 \mathrm{~min}$. The virus-sCS-E mixtures were then inoculated into BHK cells seeded in 96-well plates. After $2 \mathrm{~h}$ of incubation at $37^{\circ} \mathrm{C}$, overlay medium (DMEM containing $1 \%$ FBS and $0.5 \%$ methyl cellulose) was added to each well. The overlay medium was removed after $24 \mathrm{~h}$ of incubation at $37^{\circ} \mathrm{C}$, and the cells were washed with PBS. The cells were fixed with methanol-acetone $(1: 1, \mathrm{v} / \mathrm{v})$ for 30 
min, washed with PBS, and blocked with 1\% BSA-PBS. Cells were incubated with rabbit anti-JEV serum in 2\% BSA-PBS at room temperature for $30 \mathrm{~min}$, followed by incubation with HRP-conjugated anti-rabbit IgG antibody (Nichirei, Tokyo, Japan). Infectious foci were detected with 3,3'-diaminobenzidine (DAB; Nichirei).

Enzyme-linked immunosorbent assay (ELISA). ELISA was performed as described by Avirutnan et al. [10] with modifications. Maxi-Sorp microtiter plates (Nunc, Roskilde, Denmark) were adsorbed with soluble GAG preparations $\left(500 \mu \mathrm{g} / \mathrm{mL}\right.$ in PBS) at $4{ }^{\circ} \mathrm{C}$ overnight. After 3 washes with Tris-buffered saline containing Tween-20 (TBST), the plates were blocked with Block Ace (Yukijirushi Nyugyo Inc, Tokyo, Japan) for $2 \mathrm{~h}$ at $37^{\circ} \mathrm{C}$ and washed 3 times with TBST. Purified virus $\left(1 \times 10^{6} \mathrm{PFU} / \mathrm{mL}\right)$ in $10 \%$ FBS-TBST was added to each well and incubated for $1 \mathrm{~h}$ at $37^{\circ} \mathrm{C}$. Plates were washed 3 times with TBST and incubated for $1 \mathrm{~h}$ with rabbit anti-JEV serum in $5 \% \mathrm{BSA}-\mathrm{PBS}$ at $37{ }^{\circ} \mathrm{C}$ for $30 \mathrm{~min}$. The plates were incubated with HRP-conjugated secondary antibody (Nichirei). After 3 washes with PBS, signal was detected by adding o-phenylenediamine dihydrochloride substrate (OPD; Sigma) in citrate buffer ( $\mathrm{pH}$ 5.0). The enzymatic reaction was stopped by adding $1.25 \mathrm{M}$ $\mathrm{H}_{2} \mathrm{SO}_{4}$. The absorbance at $490 \mathrm{~nm}$ was measured by using a microplate reader (BioRad, Hercules, CA).

Flow cytometry. Cells were resuspended with cell-dissociation buffer (Invitrogen, Carlsbad, CA) and washed twice with PBS. Virus and soluble CS mixtures were incubated at $4{ }^{\circ} \mathrm{C}$ for $30 \mathrm{~min}$, added to dissociated cells, and incubated at $4{ }^{\circ} \mathrm{C}$ for $1 \mathrm{~h}$. Cells were washed twice with PBS and fixed with 1\% paraformaldehyde-PBS for 10 min. After washing 3 times with PBS, the cells were stained for 30 min with rabbit anti-JEV serum and incubated with Alexa-488-conjugated anti-rabbit IgG (Invitrogen). All labeling steps were performed on ice. Cells were analyzed with a FACS Canto system (BD Biosciences, San Diego, CA).

Real-time RT-PCR. Total RNA was extracted from cells with TRIzol (Invitrogen). One 
microgram of total RNA was reverse-transcribed by using the SuperScript ${ }^{\circledR}$ III First-Strand Synthesis system (Invitrogen) according to the manufacturer's instructions. Real-time PCR was performed with QuantiFast Probe PCR kits (QIAGEN, Valencia, CA) with the CFX96 real-time PCR detection system (BioRad). The JEV primer and probe sequences used are as follows: JEV forward, 5'-AGAACGGAAG AYAACCATGA CTAAA-3'; JEV reverse, 5'-CCGCGTTTCA GCATATTGAT-3'; and JEV probe, FAM-5'-ACCAGGAGGG CCCGG-3'-MGB [11]. Endogenous control primer and probe for mouse $\beta$-actin and 18S ribosomal RNA were purchased from Applied Biosystems (Foster City, CA).

Animal experiments. In all experiments, 17-day-old F344/NSIc rats (SLC Inc., Shizuoka, Japan) were used. Under anesthesia with isoflurane (Dainippon Pharmaceutical, Osaka, Japan), virus or a mixture of virus and sCS-E was administered intracerebrally to 2 groups of rats through the use of 27-gauge one-stop needles (Top Corp., Tokyo, Japan). At 4 days postinoculation, 3 rats from each group were sacrificed under deep anesthesia, and the brains were collected for virus titration. The viral titers of brain homogenates were determined through the use of Vero cells. At 6 days post-inoculation, the brains were collected for immunohistochemistry. All animal experimentation was performed in accordance with institutional guidelines, and ethical permission was obtained from the Hokkaido university animal care and use committee.

Immunohistochemistry. Brains were fixed in 10\% neutral-buffered formalin, embedded in paraffin wax, and sectioned at $3 \mu \mathrm{m}$. The sections were dewaxed, treated with $3 \%$ hydrogen peroxide in methanol, and blocked with 10\% normal goat serum. Rabbit anti-JEV serum was added, and the sections were incubated overnight at $4{ }^{\circ} \mathrm{C}$. The sections were washed with PBS and incubated with biotinylated goat anti-rabbit IgG (Nichirei Biosciences, Tokyo, Japan) for $10 \mathrm{~min}$ at room temperature. After further washing with PBS, the sections were incubated with streptavidin-conjugated peroxidase for $10 \mathrm{~min}$ at room temperature. The 
bound peroxidase was detected with DAB (Nichirei). The sections were counterstained with haematoxylin.

Statistical analysis. Data sets were compared by a 2-tailed, unpaired $t$-test. Statistical significance was achieved when the $p$-values were $<0.05$. Error bars indicate the standard deviation. 


\section{Results}

sCS-E inhibits JEV infection of Vero and BHK cells. Many researchers have used soluble GAGs to study the interaction between GAGs and viruses [5-7, 12]. To determine which types of CS preparations can influence JEV infection, we incubated the virus with various types of soluble CS preparations at $4{ }^{\circ} \mathrm{C}$ for $30 \mathrm{~min}$ and then inoculated Vero cells with each mixture. After $48 \mathrm{~h}$, infected cells were detected by using an indirect immunofluorescence assay for JEV antigen. Among the soluble CS preparations examined, only sCS-E effectively inhibited JEV infection (Fig. 1A). This inhibitory effect of sCS-E was also observed in the JEV infection of BHK cells (Fig. 1B). The focus reduction assay on BHK cells revealed that the level of this inhibition was dependent on the concentration of sCS-E (Fig. 1C).

sCS-E inhibits JEV binding to Vero and BHK cells. Previous studies on the relationship between GAGs and viral infection have suggested that soluble GAGs inhibit various viral infections by blocking viral entry [3-7, 13, 14]. We examined whether JEV directly interacts with sCS-E. An ELISA experiment showed that JEV significantly bound to wells precoated with sCS-E but not those bound with other soluble CS (Fig. 1D). To confirm whether sCS-E inhibited the binding of JEV to cells, a flow cytometric analysis was performed in which virus bound on the Vero cell surface at $4{ }^{\circ} \mathrm{C}$ was detected with anti-JEV serum. Preincubation of the virus with sCS-E reduced the amount of bound virus compared to mock preincubation or preincubation with other soluble CS (Fig. 1E).

sCS-E enhances JEV infection of Neuro-2a cells. Because the neuron is a major target cell in JEV infection, we investigated the effect of soluble CS treatment on the neuroblastoma cell line Neuro-2a under the same condition as was used in nonneural Vero and BHK cells. Among the soluble CS preparations examined, only sCS-E increased JEV infection (Fig. 2A-B), and it did so in a manner that was dependent on the sCS-E concentration (Fig. 2C). 
sCS-E enhances JEV replication in Neuro-2a cells. Because sCS-E had different effects on Neuro-2a cells from those on Vero or BHK cells, we investigated the influence of sCS-E on the binding of JEV to Neuro-2a cells. Flow cytometric analysis revealed that sCS-E inhibited the attachment of JEV to the Neuro-2a cell surface (Fig. 2D), as was also observed in Vero cells (Fig. 1E). To examine the effect of sCS-E treatment on the post-attachment stage, we next investigated the possible increase of viral RNA in infected cells. As shown in Fig. 2E and F, enhancement of viral RNA replication by sCS-E treatment was observed in Neuro-2a cells, but not in Vero cells. These results suggest that the sCS-E-induced enhancement of viral infection in Neuro-2a cells was attributable to enhanced viral replication rather than to increased viral attachment to the cells.

sCS-E enhances JEV infection in vivo. We next examined the effect of sCS-E on JEV infection in rat brains. Rats over 17 days old reportedly are resistant to intracerebrally administered JEV [15]. In this study, intracerebral co-inoculation of sCS-E together with JEV in 17-day-old rats resulted in a 100-fold increase in brain viral load compared to rats inoculated with JEV alone (Fig. 3A). Immunohistochemically, more JEV-positive neurons were observed in sCS-E-treated rats than rats not treated with sCS-E (Fig. 3B and C). JEV-positive cells were found in the cerebral cortex, caudate putamen, and hippocampus, and were present focally in the subventricular region, and the positive signal was almost observed in the cytoplasm of neurons (see Fig 3C, inset). 


\section{Discussion}

Our data demonstrate paradoxical effects of sCS-E on JEV infection. Although sCS-E inhibited JEV infection in nonneural Vero and BHK cells, it enhanced JEV infection in the neuroblastoma cell line Neuro-2a. In both types of cell lines, sCS-E inhibited viral entry through the inhibition of viral attachment; however, in Neuro-2a cells, it enhanced viral replication during the post-attachment stage. These results suggest that, in Neuro-2a cells, the enhancement effect of sCS-E on viral replication may overcome the inhibitory effect on viral attachment and eventually led to the enhancement of viral infection. Importantly, the enhancement effect of sCS-E on JEV infection was not confined to the neural cell line, but also was observed in vivo in rats intracerebrally co-inoculated with JEV and sCS-E.

A number of viruses reportedly utilize cell surface PGs as receptors, including human immunodeficiency virus [6], herpes simplex virus (HSV) $[3,5,7]$, and cytomegalovirus (CMV) [4]. Soluble forms of GAGs are known to inhibit the entry process of these viruses. For example, soluble heparan sulfate inhibits the HSV infection of Vero, HepG-2, CHO, and mouse L cells [13], and it also inhibits the CMV infection of human foreskin fibroblasts and human embryonic lung fibroblasts [14]. Flaviviruses, such as JEV, yellow fever virus, West Nile virus, Murray Valley encephalitis (MVE), and St. Louis encephalitis virus, have variable affinities for GAGs; this property contributes to viral attachment to host cells [8, 12, 16-21]. The high affinity of an attenuated JEV strain for heparan sulfate is known to be responsible for its reduced virulence after peripheral inoculation, due to the rapid clearance of the virus from the bloodstream by the reticuloendothelial system [8]. A previous study on the antiviral activity of sCS-E on DENV infection demonstrated that sCS-E inhibits JEV infection in BHK cells and mentioned the possibility of its use as an antiviral drug [22].

In our study, sCS-E inhibited JEV entry at an early stage of infection. However, the potential use of sCS-E as an antiviral agent against JEV infection should be approached with 
caution because, although sCS-E inhibited JEV infection in nonneural cells (e.g., Vero and BHK cells), it enhanced viral replication in a neural cell line and in brain, the main targets of JEV. Studies on the relationship between GAGs and virus infection may need to include a study of the effects of GAGs in natural target cells and organs.

The precise mechanism for the enhancement of viral replication remains uncertain. One possibility is that this enhancement effect involves the suppression of the innate immune response, which is a major host defense mechanism against viral infection in neural cells. In fact, we found that the high-level of constitutive IFN- $\alpha$ expression observed in Neuro-2a cells was inhibited by sCS-E treatment (data not shown).

In conclusion, our data show that sCS-E has paradoxical effects on JEV infection depending on the cell type. A trial use of sCS-E as an antiviral drug against JEV infection may require special attention be given to its effects in the neuron, the major target of JEV.

\section{Acknowledgement}

We thank Ms. Satoko Ueno for technical assistance. This work was supported in part by a Grant-in-Aid for Challenging Exploratory Research (TK), Scientific Research B (23390016) (KS), and the Program of Founding Research Centers for Emerging and Reemerging Infectious Diseases (TK, MO, HS, TM, and DF) administered by the Ministry of Education, Culture, Sports, Science and Technology, Japan. 


\section{References}

[1] J. Kimura-Kuroda, M. Ichikawa, A. Ogata, K. Nagashima, K. Yasui, Specific tropism of Japanese encephalitis virus for developing neurons in primary rat brain culture., Arch Virol 130 (1993) 477-484.

[2] M. Ishii, N. Maeda, Oversulfated chondroitin sulfate plays critical roles in the neuronal migration in the cerebral cortex., J Biol Chem 283 (2008) 32610-32620.

[3] K. Bergefall, E. Trybala, M. Johansson, T. Uyama, S. Naito, S. Yamada, H. Kitagawa, K. Sugahara, T. Bergström, Chondroitin sulfate characterized by the E-disaccharide unit is a potent inhibitor of herpes simplex virus infectivity and provides the virus binding sites on gro2C cells., J Biol Chem 280 (2005) 32193-32199.

[4] T. Compton, D. Nowlin, N. Cooper, Initiation of human cytomegalovirus infection requires initial interaction with cell surface heparan sulfate., Virology 193 (1993) 834-841.

[5] E. Lycke, M. Johansson, B. Svennerholm, U. Lindahl, Binding of herpes simplex virus to cellular heparan sulphate, an initial step in the adsorption process., J Gen Virol 72 ( Pt 5) (1991) 1131-1137.

[6] M. Patel, M. Yanagishita, G. Roderiquez, D. Bou-Habib, T. Oravecz, V. Hascall, M. Norcross, Cell-surface heparan sulfate proteoglycan mediates HIV-1 infection of T-cell lines., AIDS Res Hum Retroviruses 9 (1993) 167-174.

[7] M. Shieh, D. WuDunn, R. Montgomery, J. Esko, P. Spear, Cell surface receptors for herpes simplex virus are heparan sulfate proteoglycans., J Cell Biol 116 (1992) 1273-1281.

[8] E. Lee, R. Hall, M. Lobigs, Common E protein determinants for attenuation of glycosaminoglycan-binding variants of Japanese encephalitis and West Nile viruses., J Virol 78 (2004) 8271-8280. 
[9] C. Aoki, K.I. Hidari, S. Itonori, A. Yamada, N. Takahashi, T. Kasama, F. Hasebe, M.A. Islam, K. Hatano, K. Matsuoka, T. Taki, C.T. Guo, T. Takahashi, Y. Sakano, T. Suzuki, D. Miyamoto, M. Sugita, D. Terunuma, K. Morita, Y. Suzuki, Identification and characterization of carbohydrate molecules in mammalian cells recognized by dengue virus type 2., J Biochem 139 (2006) 607-614.

[10] P. Avirutnan, L. Zhang, N. Punyadee, A. Manuyakorn, C. Puttikhunt, W. Kasinrerk, P. Malasit, J.P. Atkinson, M.S. Diamond, Secreted NS1 of dengue virus attaches to the surface of cells via interactions with heparan sulfate and chondroitin sulfate E., PLoS Pathog 3 (2007) e183.

[11] K. Shirato, H. Miyoshi, H. Kariwa, I. Takashima, Detection of West Nile virus and Japanese encephalitis virus using real-time PCR with a probe common to both viruses., J Virol Methods 126 (2005) 119-125.

[12] M. Lobigs, I. Marshall, R. Weir, L. Dalgarno, Genetic differentiation of Murray Valley encephalitis virus in Australia and Papua New Guinea., Aust J Exp Biol Med Sci 64 ( Pt 6) (1986) 571-585.

[13] S. Gruenheid, L. Gatzke, H. Meadows, F. Tufaro, Herpes simplex virus infection and propagation in a mouse L cell mutant lacking heparan sulfate proteoglycans., J Virol 67 (1993) 93-100.

[14] J. Neyts, R. Snoeck, D. Schols, J. Balzarini, J. Esko, A. Van Schepdael, E. De Clercq, Sulfated polymers inhibit the interaction of human cytomegalovirus with cell surface heparan sulfate., Virology 189 (1992) 48-58.

[15] A. Ogata, K. Nagashima, W. Hall, M. Ichikawa, J. Kimura-Kuroda, K. Yasui, Japanese encephalitis virus neurotropism is dependent on the degree of neuronal maturity., $\mathrm{J}$ Virol 65 (1991) 880-886. 
[16] C. Hahn, J. Dalrymple, J. Strauss, C. Rice, Comparison of the virulent Asibi strain of yellow fever virus with the 17D vaccine strain derived from it., Proc Natl Acad Sci U S A 84 (1987) 2019-2023.

[17] D. Kobiler, S. Lustig, Y. Gozes, D. Ben-Nathan, Y. Akov, Sodium dodecylsulphate induces a breach in the blood-brain barrier and enables a West Nile virus variant to penetrate into mouse brain., Brain Res 496 (1989) 314-316.

[18] E. Lee, M. Lobigs, Substitutions at the putative receptor-binding site of an encephalitic flavivirus alter virulence and host cell tropism and reveal a role for glycosaminoglycans in entry., J Virol 74 (2000) 8867-8875.

[19] M. Lobigs, R. Usha, A. Nestorowicz, I. Marshall, R. Weir, L. Dalgarno, Host cell selection of Murray Valley encephalitis virus variants altered at an RGD sequence in the envelope protein and in mouse virulence., Virology 176 (1990) 587-595.

[20] C. Mandl, H. Kroschewski, S. Allison, R. Kofler, H. Holzmann, T. Meixner, F. Heinz, Adaptation of tick-borne encephalitis virus to BHK-21 cells results in the formation of multiple heparan sulfate binding sites in the envelope protein and attenuation in vivo., $\mathrm{J}$ Virol 75 (2001) 5627-5637.

[21] T. Monath, C. Cropp, G. Bowen, G. Kemp, C. Mitchell, J. Gardner, Variation in virulence for mice and rhesus monkeys among St. Louis encephalitis virus strains of different origin., Am J Trop Med Hyg 29 (1980) 948-962.

[22] D. Kato, S. Era, I. Watanabe, M. Arihara, N. Sugiura, K. Kimata, Y. Suzuki, K. Morita, K.I. Hidari, T. Suzuki, Antiviral activity of chondroitin sulphate E targeting dengue virus envelope protein., Antiviral Res 88 (2010) 236-243. 


\section{Figure Legends}

Fig. 1. Effect of CS on JEV infection in Vero and BHK cells. (A and B) JEV was incubated with various CS preparations (final concentration of $25 \mu \mathrm{g} / \mathrm{mL}$ ), and Vero (A) and BHK (B) cells were infected at a multiplicity of infection (MOI) of 0.5 . The same concentration of soluble CS was maintained throughout a $20-\mathrm{h}$ (BHK) or 48-h (Vero) incubation. Infected cells were detected with an indirect immunofluorescence assay. (C) JEV ( $\approx 40$ FFU) was incubated with various concentrations of SCS-E (final concentrations of $0,0.1,1,10$, and 100 $\mu \mathrm{g} / \mathrm{mL}$ ), and BHK cells were infected. Viral infectivity was determined by a focus reduction assay compared with control (JEV only). Asterisk indicates significant difference compared to control as analyzed by 2 -tailed $t$-test (**, $p<0.01$; and $* * *, p<0.001)$. (D) Microtiter plates were coated with various types of soluble CS and heparin preparations. After JEV was added to each well, bound virus was detected through the use of anti-JEV serum and HRP-conjugated anti-rabbit IgG, followed by colorization with OPD. Asterisk indicates significant difference compared to control (PBS-coated wells, dotted line) as analyzed by 2-tailed $t$-test $(p<0.05)$. (E) Vero cells were incubated at $4{ }^{\circ} \mathrm{C}$ for $1 \mathrm{~h}$ with JEV or with a preincubated mixture of JEV (MOI 5) and $50 \mu \mathrm{g} / \mathrm{mL}$ sCS-A through sCS-E. Viral binding was detected by flow cytometry using anti-JEV serum and a fluorescent secondary antibody. Flow cytometric histograms of virus-bound cells (black line histogram) were overlaid with histograms of mock-infected cells (gray filled histogram).

Fig. 2. Effect of CS on JEV infection in Neuro-2a cells. (A and B) JEV was incubated with various CS preparations (final concentration of $25 \mu \mathrm{g} / \mathrm{mL}$ ), and Neuro-2a cells were infected at an MOI of 0.5 . The same concentration of soluble CS was maintained throughout the 48-h incubation. Infected cells were detected with an indirect immunofluorescence assay (A), and the percentage of infected cells was determined with MetaMorph software (B). (C) JEV was 
incubated with various concentrations of CS-E (final concentrations of $0,0.25,2.5$, and 25 $\mu \mathrm{g} / \mathrm{mL}$ ) and used to infect Neuro-2a cells at an MOI of 0.5 . After $48 \mathrm{~h}$, the percentage of infected cells was determined by using an indirect immunofluorescence assay and MetaMorph software. Asterisks indicate significant differences compared to control group (JEV only; untreated), as analyzed by 2-tailed $t$-test $(p<0.05)$. (D) Neuro-2a cells were incubated at $4{ }^{\circ} \mathrm{C}$ for $1 \mathrm{~h}$ with JEV or a preincubated mixture of JEV (MOI 150) and 50 $\mu \mathrm{g} / \mathrm{mL}$ of sCS-A through sCS-E. Viral binding was detected by flow cytometry with anti-JEV serum and a fluorescent secondary antibody. Flow cytometric histograms of virus-bound cells (black line histogram) were overlaid with histograms of mock-infected cells (gray filled histogram). (E and F) JEV only or a preincubated mixture with sCS-E was inoculated in Neuro-2a cells (F) and Vero cells (E) at an MOI of 0.5. After a 1-h inoculation, the viral inoculum was removed. The cells were washed with PBS, followed by maintenance medium with or without sCS-E $(25 \mu \mathrm{g} / \mathrm{mL})$ (open square, JEV only; filled square, JEV with sCS-E). The cell lysate was harvested at $0 \mathrm{~h}$ (immediately after the wash with PBS) and at the indicated time points, and RNA was extracted and subjected to a 2-step real time RT-PCR. After normalization for the expression of actin mRNA (Neuro-2a) or ribosomal RNA (Vero), the fold increase of viral RNA was determined relative to that of viral RNA in $0 \mathrm{~h}$ post infection. Asterisks indicate significant differences between control group (JEV only) and test group (JEV with sCS-E), as analyzed by 2 -tailed $t$-test $(*, p<0.05$; and $* *, p<0.01$ ).

Fig. 3. Effect of sCS-E on JEV infection in vivo. (A) Seventeen-day-old rats were intracerebrally inoculated with JEV $\left(3 \times 10^{6} \mathrm{PFU} /\right.$ rat $)$ in the presence or absence of sCS-E (7.5 $\mu \mathrm{g} / \mathrm{rat})$. At 4 days postinoculation, the brains were collected, and the virus levels were titrated on Vero cells. Asterisks indicate significant differences compared to the control group, as analyzed by 2-tailed $t$-test $(n=3, p<0.05)$. (B and C) Distribution of JEV antigen in the 
cerebral cortex of rats at 6 days postinoculation. Brains were collected and processed for immunohistochemistry. Staining with an anti-JEV antibody demonstrated that there were more JEV-positive neurons in sCS-E-treated rats (C) than in untreated rats (B). The insets show high magnification of neurons. Scale bars represent $200 \mu \mathrm{m}$ (B and C), and $25 \mu \mathrm{m}$ (insets). 
A

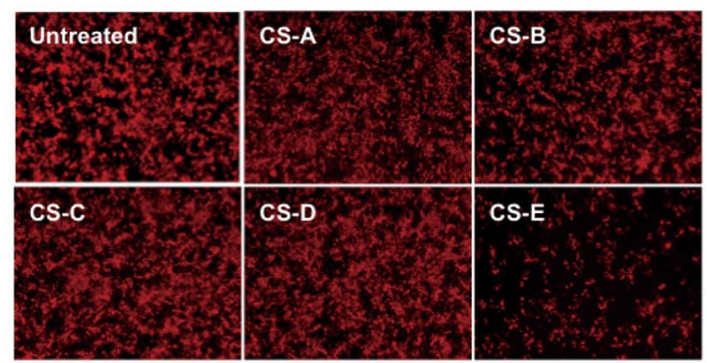

B

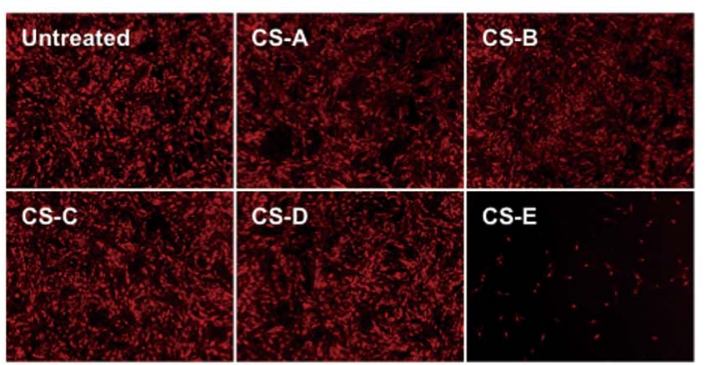

C
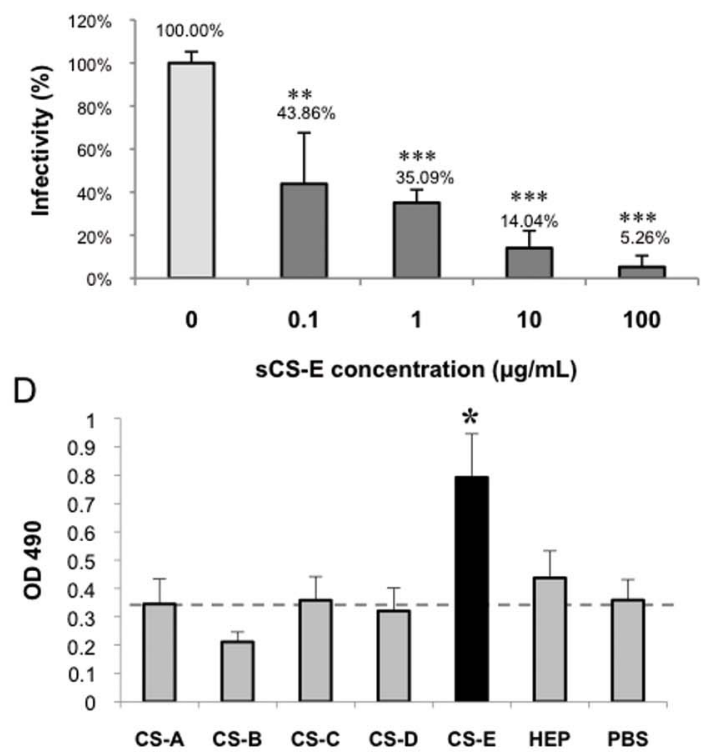

E
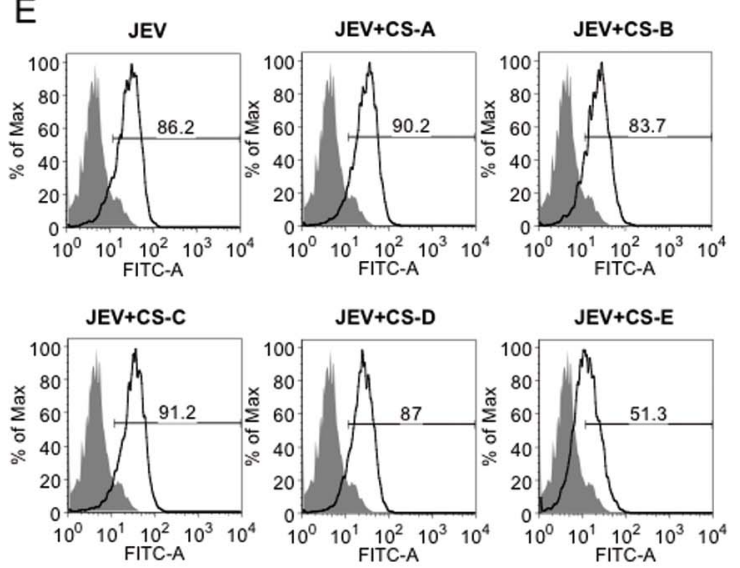
A

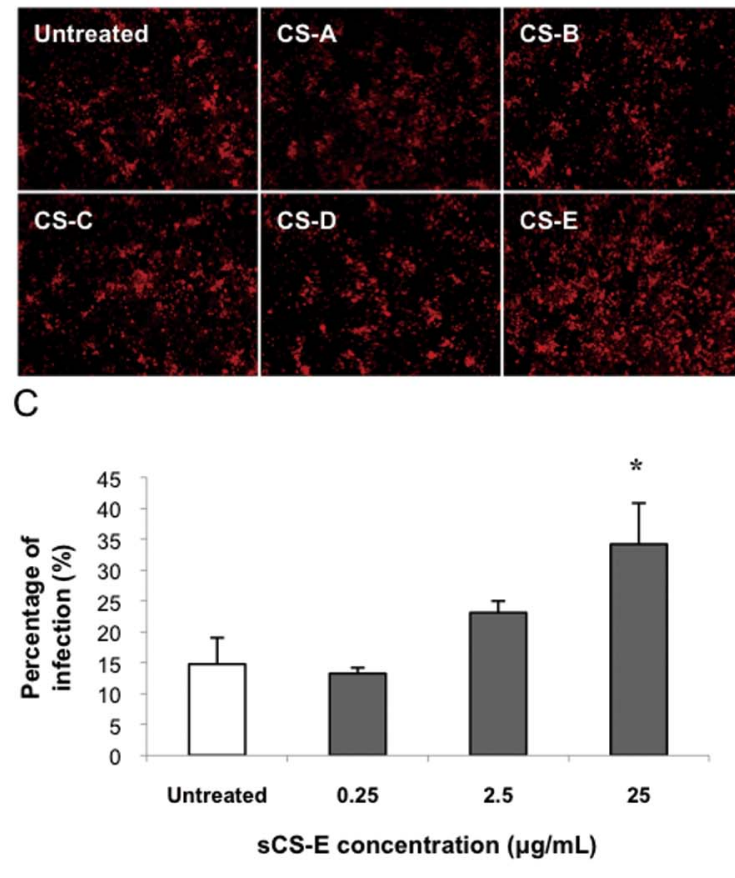

E

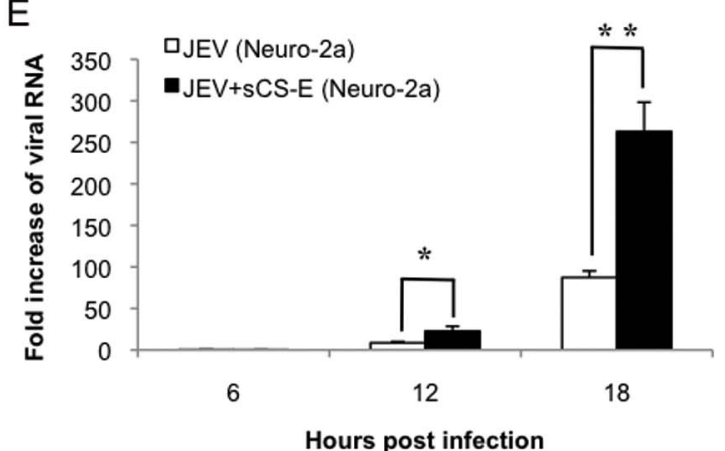

B

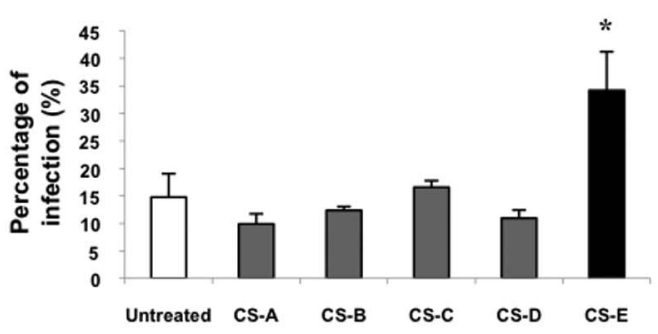

D
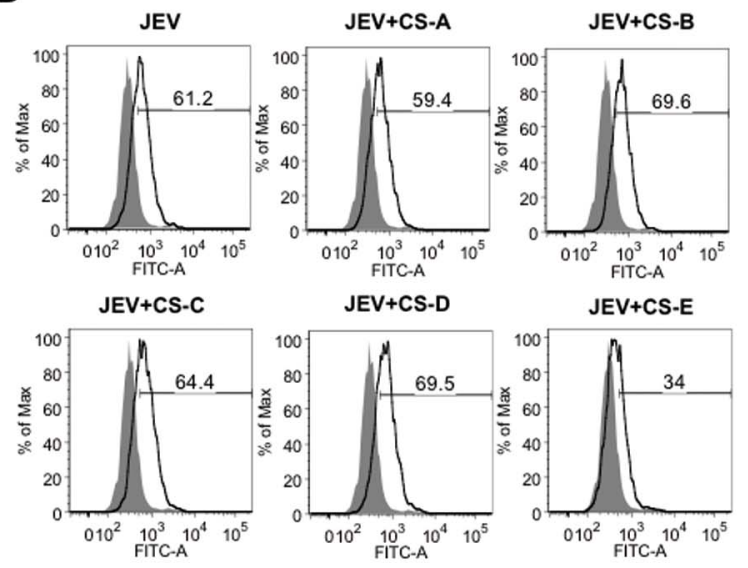

F

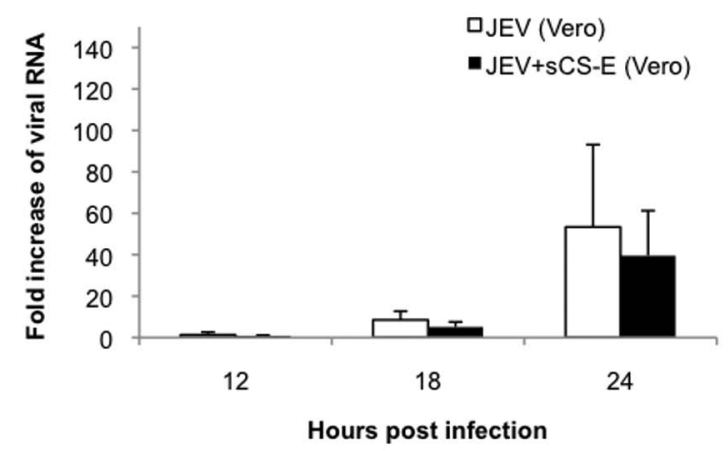


A

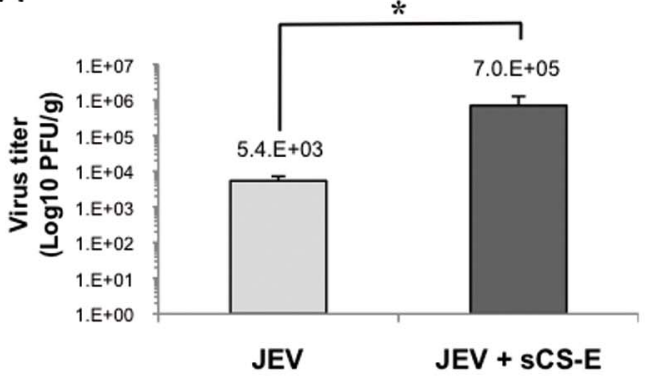

B

JEV

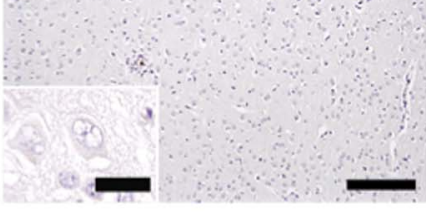

C

JEV + sCS-E

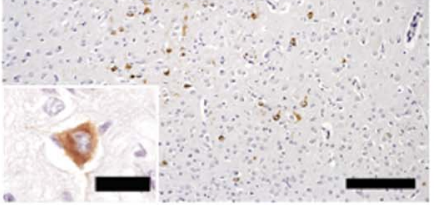

